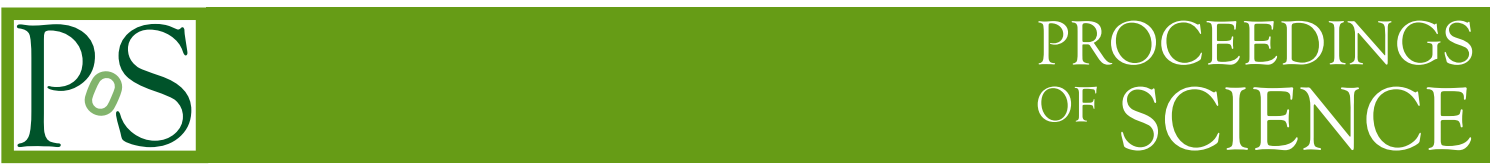

\title{
LHCb ECAL upgrade II
}

\section{Sergei Kholodenko on behalf of the LHCb ECAL UII working group}

NRC «Kurchatov Institute» - IHEP, 1 Nauki sq, Protvino, Russia

E-mail: sergey.kholodenko@cern.ch

The aim of the Phase-2 Upgrade of LHCb is to collect up to $300 \mathrm{fb}^{-1}$ of data in a few years, operating at luminosity of $1.5 \times 10^{34} \mathrm{~cm}^{-2} \mathrm{~s}^{-1}$. Because of the significant increase in particle densities and radiation doses, the present $\mathrm{LHCb}$ Electromagnetic Calorimeter (ECAL) will require a major revision. The increased instantaneous and integrated luminosity will result in very high particle density and radiation doses in the areas close to the beam pipe. In these conditions, ECAL has to provide high-quality energy and position measurement for electromagnetic showers, as well as separation of two closely lying showers. Another requirement for the whole ECAL, which is aimed to reduce combinatorial background at high luminosity operation, is the ability to measure the time of arrival of the photon or electron with an accuracy of few tens of picoseconds. The intrinsic time resolution of the ECAL modules is expected to be sufficient to meet this requirement, although the use of an additional timing layer is not excluded. The expected particle flow and radiation doses strongly depend on the distance from the beam pipe and determine the technology and granularity of the upgraded ECAL modules. The upgraded ECAL will be subdivided accordingly into several zones. The central part, with the highest expected doses, will be a sampling spaghetti calorimeter (SPACAL) based on radiation-hard crystal scintillators and a tungsten absorber. The peripheral areas will be instrumented with modified Shashlik type modules, similar to the modules of the present ECAL, with modifications aiming to achieve the best time resolution for this technology. The intermediate part will be a spaghetti calorimeter with polystyrene-based scintillating fibres and a moulded lead absorber. The main advantages of using lead-polystyrene spaghetti type are the possibility to modify granularity with minimal intervention and easily replace fibres damaged by radiation.

An extensive R\&D campaign is ongoing to optimize the Upgrade 2 ECAL structure. It includes:

- studies of scintillating materials, in terms of scintillation kinetics and radiation hardness;

- simulation studies to find the optimal detector layout, longitudinal segmentation and granularity;

- beam test studies of the performance of various ECAL module prototypes, both for central (SPACAL) and peripheral areas.

For the moment, a time resolution for $5 \mathrm{GeV}$ electrons achieved for W-Crystal and Lead-Polystyrene Spacal prototypes is about 20 ps, and better than 40 ps for Shashlik type modules.

\footnotetext{
*** Particles and Nuclei International Conference - PANIC2021 ***

*** 5 - 10 September, $2021 * * *$

$* * *$ Online $* * *$
} 


\section{Introduction}

The LHCb experiment [1] will have to be upgraded to operate during the HL-LHC [2], i.e. after Long Shutdown 4 (LS4). The goal of collecting an integrated luminosity of $300 \mathrm{fb}^{-1}$ in Upgrade Phase II implies that the calorimeter must be capable of sustaining integrated radiation doses of up to $1 \mathrm{MGy}$ in the innermost modules (Figure 1, left). The $40 \mathrm{kGy}$ boundary, shown as a bold black line, represents the limit for the Shashlik [3], motivating studies of the alternative radiation hard technologies.
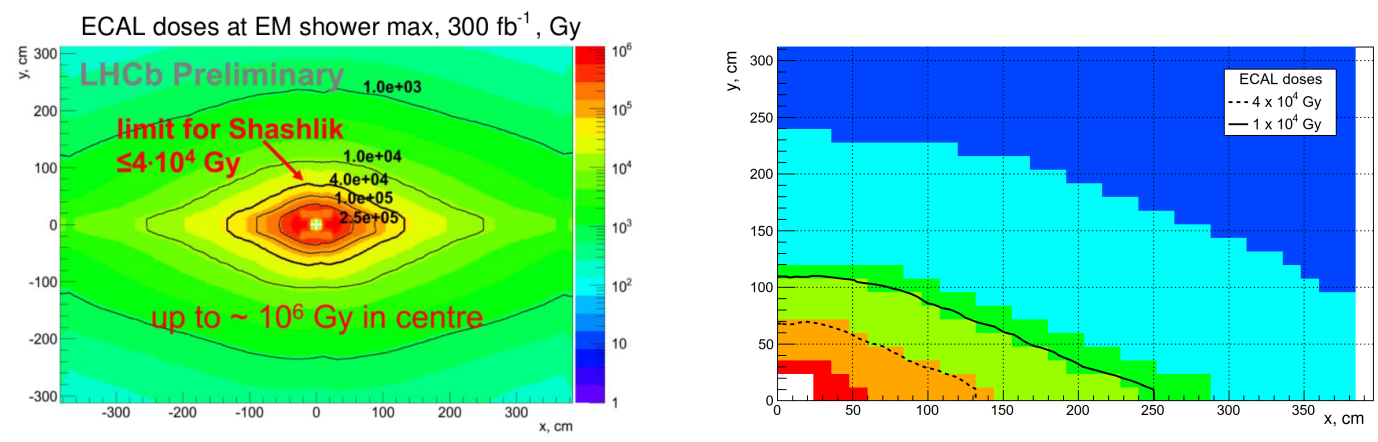

Figure 1: Integrated radiation dose (left) and baseline ECAL UII regions (right). Different colors represent different cell sizes (from red $-15 \times 15 \mathrm{~mm}^{2}$ to blue $-120 \times 120 \mathrm{~mm}^{2}$.

The baseline for the inner region is so-called SPAghetti CALorimeter (SPACAL) [4]. SPACAL type modules provide flexibility in defining the cell size, hence this region may also be upgraded at a later stage. For the innermost modules the current baseline considers tungsten absorber and radiation hard crystal fibres, e.g. GAGG, acting both as scintillator and light-transporting medium, with a cell size of $15 \times 15 \mathrm{~mm}^{2}$. For the intermediate region, where Shashlik is not usable, but expected integrated radiation dose for $300 \mathrm{fb}^{-1}$ is not exceeding $200 \mathrm{kGy}$, a SPACAL technology with a lead-based absorber equipped with polystyrene plastic scintillating fibres is considered. The rest $\sim 95 \%$ of the area to be covered with the modified Shashlik modules of different cell sizes. The proposed technologies, granularities and number of modules per region are presented in the Table 1.

\begin{tabular}{lclrr} 
Region & Cell size $\left[\mathrm{mm}^{2}\right]$ & Module type & \#modules & \#cells \\
\hline \hline 1 & $15 \times 15$ & SPACAL W + crystal fibre (up to 1 MGy) & 32 & 2048 \\
2 & $30 \times 30$ & SPACAL Pb + plastic fibre (up to 200 kGy) & 144 & 2304 \\
3 & $40 \times 40$ & New + refurbished Shashlik (up to 40 kGy) & 448 & 4032 \\
4 & $60 \times 60$ & Refurbished and rebuilt Shasklik & 1344 & 5376 \\
5 & $120 \times 120$ & Refurbished Shaslik & 1344 & 1344 \\
\hline Total & & & 3312 & 15104
\end{tabular}

Table 1: Cell size, technology and number of modules per region proposed for the ECAL upgrade II. The regions correspond to the differently coloured zones in Figure 1 (right) from region 1 (red) to 5 (blue). 


\section{SPACAL: W-Crystal}

The Crystal-based SPACAL module consists of 3D-printed tungsten absorber and $1 \times 1 \mathrm{~mm}^{2}$ crystal fibres, longitudinally splitted in two parts: 7 radiation lengths $\left(X_{0}\right)$ for the front part and $18 X_{0}$ (back part), as it shown on the Figure 2 left. Time resolution as a function of incident electron energy was measured with the Test Beam at DESY (Hamburg) and shown on the Figure 2 (right). This result was achieved with R7600U-20 PMTs directly coupled to crystal fibres.
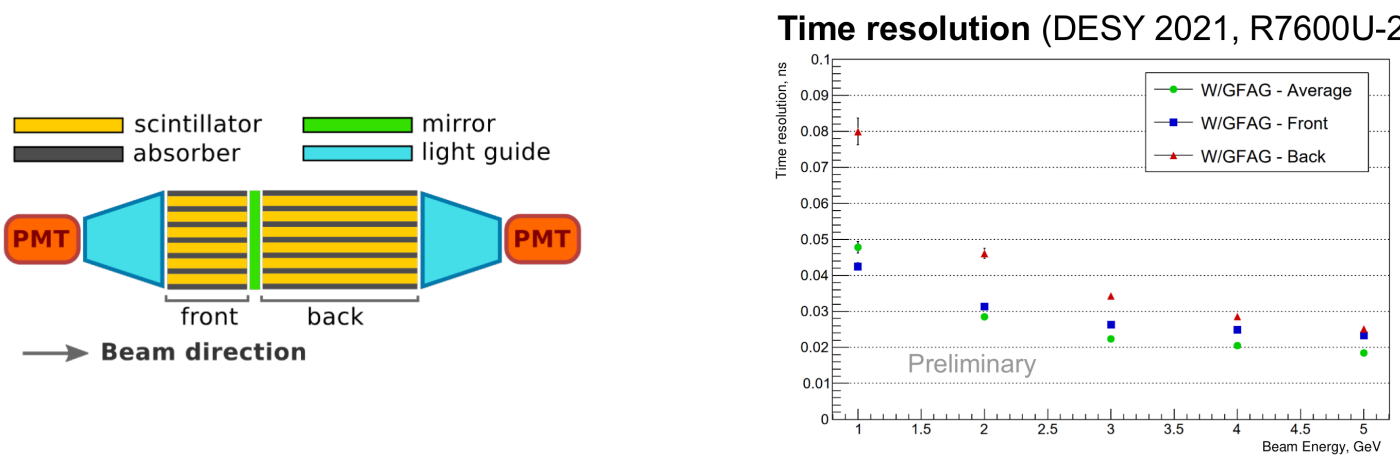

Figure 2: SPACAL W-Crystal prototype

The energy resolution measurements were done for the single-cell $30 \times 30 \mathrm{~mm}^{2}$ prototype read by R12421 PMTs through lightguides (Figure 2 left). The preliminary results gives $10.6 \%$ of stochastic term and $1.9 \pm 0.5 \%$ of constant term. But the scintillation decay time of radiation hard crystals still to be improved - the "fastest" one, GFAG, has a decay time $\tau \sim 60 \mathrm{~ns}$ [5].

\section{SPACAL: Lead-Polysterene}

The module comprises the molded lead-based absorber $(\mathrm{Pb} 84 \%)$ and scintillating fibres. In addition to adjustable granularity, this design allows replacement of fibres damaged by radiation, that could be performed during the annual stops. A single cell prototype was produced, with dimensions $30.25 \times 30.25 \mathrm{~mm}^{2}$ and 121 fibres. It was tested at the CERN SPS electron beam in August 2021. The light was read out with Hamamatsu® R11187 PMTs from both front and back sides through the $50 \mathrm{~mm}$ long lightguides. The single cell schematics and photo of the prototype are shown in the Figure 3.
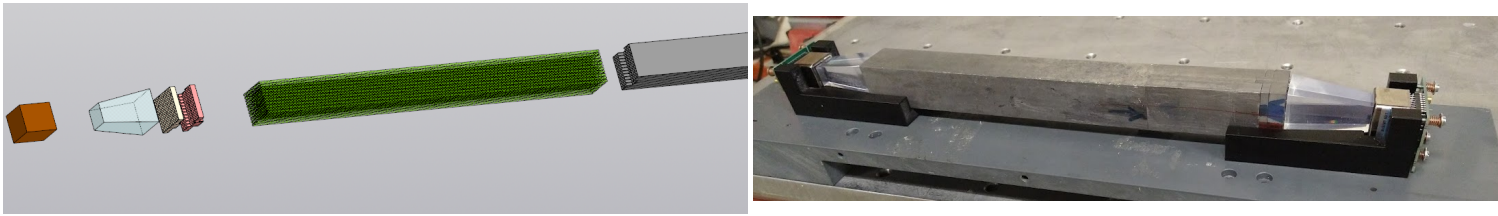

Figure 3: SPACAL: Lead-Polysterene schematic view (left) and photo (right).

During the beam-tests fibres of two producers were tested: Kuraray SCSF-78 and fibres produced in NRC «Kurchatov Institute» - IHEP (Protvino). Both fibres have $\oslash 2 \mathrm{~mm}$ diameters. Despite the factor of 2 in light yield (due to some problems with cladding) the time resolution 
results for high energy electrons are comparable and presented in Figure 4 with orange and violet colours for SCSF-78 and Protvino fibres respectively.

\section{Modified Shashlik}

The Shashlik part of R\&D includes a test of a few modules (similar to the present ECAL) modified to improve performance in terms of time resolution. The modification includes new WLS fibres (with shorter scintillation decay time, e.g. Kuraray YS2 and YS4), a double-sided readout scheme and PMTs with a small transit time spread (R7600U-20). The possibility of using combined time [6] (weighed average time of signal arrival between front and back PMTs) allows to reach time resolution $\sigma<50$ ps for electrons with $E>5 \mathrm{GeV}$. Additional advantage of fast WLS fibres shorter pulses to fit the $25 \mathrm{~ns}$ bunch-crossing period, are illustrated on the Figure 4 left. On the right side of the same Figure a distribution of combined time $\left(T_{\text {comb }} \approx 0.75 \cdot T_{\text {Back }}+0.25 \cdot T_{\text {Front }}\right)$ with reference to $\mathrm{T} 0\left(\sigma_{T_{0}}=13 \pm 1 \mathrm{ps}\right)$ for electrons with $\mathrm{E}=100 \mathrm{GeV}$ is shown.
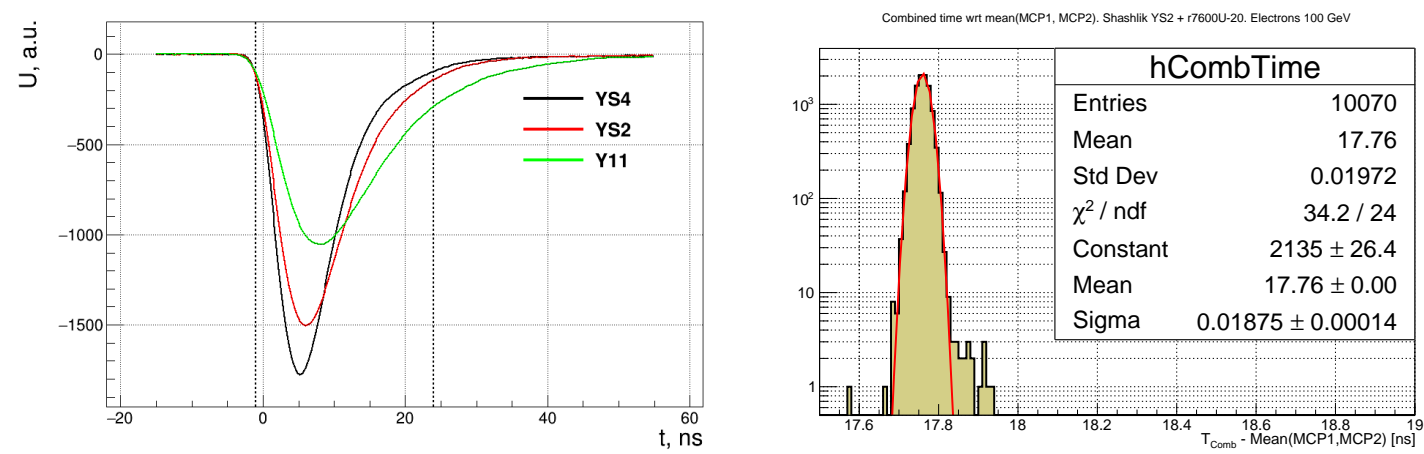

Figure 4: Output Pulse shapes for the shashlik modules equipped with Y11 (green), YS2 (red) and YS4 (black) fibres (left) and distribution of combined time for the Shashlik module equipped with YS2 fibres and two R7600U-20 PMTs for incident electrons with $\mathrm{E}=100 \mathrm{GeV}$ (right).

The time resolution of both Shashlik and Lead-Polysterene SPACAL prototypes as a function of incident electron energy in the range of $1-100 \mathrm{GeV}$ is presented in the Figure 5.

The time resolution $\left(\sigma_{T}\right)$ of single cell Shashlik module equipped with Kuraray YS2 fibres and R7600U-20 PMTs from both front and back sides could be estimated with the function:

$$
\sigma_{T}=\frac{86 p s \cdot G e V^{1 / 2}}{\sqrt{E}} \oplus 16 p s
$$

\section{Summary}

The proposed baseline plan for LS3 consists of replacing the inner region $(32+144$ modules) with SPACAL type equipped with plastic fibres. Keeping in mind that innermost 32 modules to be replaced with radiation hard crystals at LS4. The plan includes also rearranging of the existing Shashlik modules according to the occupancy map, as shown in Figure 1 (right). 


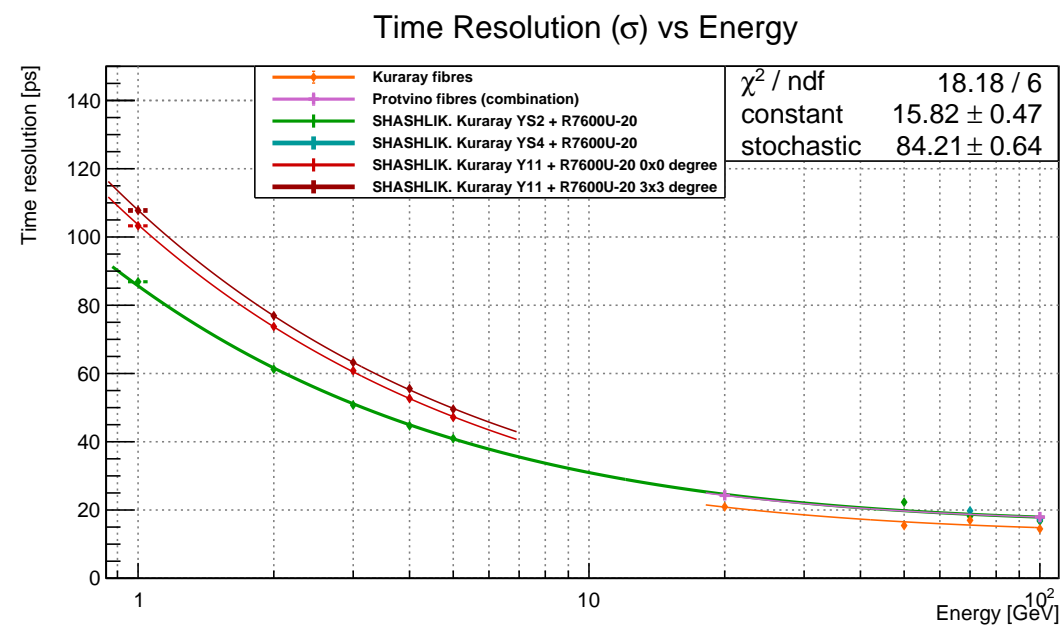

Figure 5: Time resolution (sigma) as a function of incident electron energy for different Shashlik modules (with Y11, YS2 and YS4 fibres) and Spaghetti module produced with molded Pb-based absorber equipped with $\oslash 2 \mathrm{~mm}$ scintillating fibres (Kuraray SCSF-78 and Protvino).

Introducing the additional timing layer made with either Silicon or Micro-Channel Plate (LAPPD [7]) detector are also pursued. Such layer placed at the shower maximum (after $7 X_{0}$ ) inside the ECAL module provides precise $(\sigma<20 \mathrm{ps})$ time measurements even for the low energetic showers.

\section{Acknowledgements}

I would like to thank A. Barnyakov and M .Barnyakov for their kind assistance in getting MCP-PMTs used as a time reference, V. Rykalin and Kuraray for the preproduction fibres samples.

The measurements leading to these results have been performed at the Test Beam Facility at DESY Hamburg (Germany), a member of the Helmholtz Association (HGF) [8], and CERN North Area Test Beams Facility.

\section{References}

[1] A. A. Alves, Jr. et al. [LHCb], JINST 3 (2008), S08005

[2] G. Apollinari, I. Béjar Alonso, O. Brüning, M. Lamont and L. Rossi, CERN-2015-005

[3] [LHCb], CERN-LHCC-2000-036

[4] G. Dosovitskiy, et al. Nucl. Instrum. Meth. A 999 (2021), 165169

[5] L. Martinazzoli, et al. Nucl. Instrum. Meth. A 1000 (2021), 165231

[6] Y. Guz [LHCb and Crystal Clear], JINST 15 (2020) no.09, C09046

[7] M.J. Minot, et al. Nucl. Instrum. Meth. A 936 (2019), 527-531

[8] R. Diener, et al. Nucl. Instrum. Meth. A 922 (2019), 265-286 